\title{
Torsion topological groups with minimal open sets
}

\author{
M.C. Thornton
}

Torsion topological groups with the additional property that the intersection of open sets is open are considered and their topological structure is determined:

1) the topology is uniquely determined by a normal subgroup;

2) each group is uniquely an extension of an indiscrete group by a discrete group;

3) the topology may be changed within limits without changing the dual group.

An A-space is a topological space where the intersection of open sets is open ([1], [5], [8]). For a point $x$ in an $A$-space $X, U_{x}$, the minimal open set containing $x$, is the intersection of all open sets containing $x$. Topological groups and semi-topological groups are defined as in [3]. $G_{e}$ will denote the component of the identity $e$.

LEMMA 1. Let $G$ be an A-space semi-topological group with $a, b \in G$. Then $a U_{b}=U_{a b}=U_{a} b$.

Proof. $U_{a} b$ is open and contains $a b$ so $U_{a b} \subset U_{a} b$. Also $U_{b} \subset a^{-1} U_{a b}$ so $a U_{b} \subset U_{a b}$. Thus $U_{a b}=a U_{b}$ and likewise $U_{a b}=U_{a} b$.

The next lemma is an extension of the results in [6].

LEMMA 2. Let $G$ be a semi-topological torsion group with an

Received 5 January 1971. 
A-space topology. Then $U_{e}$ is an open and closed normal subgroup, has the indiscrete topology induced on it, and is $G_{e}$. Further, $G$ is a topological group whose minimal open sets are the cosets of $U_{e}$.

Proof. If $g \in U_{e}$ then $g U_{e}=U_{g} \subset U_{e}$ thus $U_{e} U_{e} \subset U_{e}$. But $U_{e} U_{e}$ is open so that $U_{e} \subset U_{e} U_{e}$ and $U_{e} U_{e}=U_{e}$. Since $g$ has finite order, $g^{-1}$ is a power of $g$ so $g^{-1} \in U_{e}$. Therefore $U_{e}$ is a subgroup. Since $U_{e} x=U_{x}=x U_{e}, x U_{e} x^{-I}=U_{e}$ and so $U_{e}$ is normal.

Suppose $V$ is a proper open subset of $U_{e}$ containing $g$. Then $g^{-1} V \subset U_{e}$ is an open subset of $U_{e}$ containing $e$ with $g^{-1} V \neq U_{e}$. This contradicts the minimality of $U_{e}$ so $V$ cannot exist and so $U_{e}$ is indiscrete. Since $U_{e}$ is open it is also closed $[2,5.5]$. Since $U_{e}$ is connected, $U_{e} \subset G_{e}$. But $G_{e}-U_{e}$ is open since $U_{e}$ is closed. Thus the connectedness of $G_{e}$ implies $U_{e}=G_{e}$. The homogeneity of $G$ shows the cosets of $U_{e}$ are a base for the topology. By [2, 4.5] $G$ is a topological group.

LEMMA 3. Let $H$ be a normal subgroup of a group $G$. Define a subset of $G$ to be open iff it is a union of H-cosets. Then $G$ is a topological group with on A-space topology and $G_{e}=H$.

Proof. Since $H$-cosets are disjoint and exhaust $G$ they form a base for a topology. In this topology $H$ is the component of the identity. $H$ with the induced topology is an indiscrete topological group. To show $G$ is a topological group we verify $\mu: G \times G \rightarrow G$ given by $\mu(g, k)=g^{-I_{k}}$ is continuous. It suffices to show $\mu\left(U_{g} \times U_{k}\right)=U_{g^{-1}}=g^{-I_{k H}}$ since $g^{-I_{k H}}$ is the smallest open set containing $g^{-I_{k}}$. iLet $g h \in g H=U_{g}$. Then

$$
\mu\left(g h \times U_{k}\right)=(g h)^{-1} U_{k}=h^{-1} g^{-1}(k H)=g^{-1} k\left(h^{\prime} H\right)=g^{-I_{k H}}=U_{g^{-1}} .
$$


Thus

$$
\mu\left(U_{g} \times U_{k}\right)=\mu\left(g H \times U_{k}\right)=\underset{h \in H}{U} \mu\left(g h \times U_{k}\right)=U_{g}^{-I_{k}} .
$$

From the above it is clear that any finite topological space $X$ which is homogeneous can carry a group structure making it a topological group. For example if $X$ has $p$ minimal open sets with $q$ points in each, then $X$ can be given the group structure of $z_{p} \oplus z_{q}$ or $z_{p q}$.

Lemmas 2 and 3 prove the following theorem which extends [2, 4.21 (b)] to $A$-spaces. It also gives information on the number of topologies possible on a finite set, a problem considered in [4]. As in [6], the corollary gives an exact answer to a special case of the problem considered in $[7]$.

THEOREM 4. There is a bijective correspondence between normal subgroups of a torsion group $G$ and the A-space topologies on $G$ giving a topological group.

COROLLARY 5. A finite topological group has exactly $2^{r}$ open subsets where $r$ is the index of the largest connected subgroup.

Proof. A base for the topology is given by the $r$ disjoint open subsets which are the cosets of the largest connected subgroup, the component of the identity. This result was implicit in [6].

Let $G$ be an $A$-space torsion topological group (not assumed abelian). Let $S$ denote the circle group with the usual topology. The dual of $G$ (or character group) is defined by

$$
G^{*}=\operatorname{Hom}_{c}(G, S)=\text { the set of continuous homomorphisms from } G \text { to } S \text {. }
$$

$G^{*}$ with the compact-open topology and the abelian group structure defined

by $\left(f_{1}+f_{2}\right) g=f_{1}(g) f_{2}(g)$ is a topological group ([3, Section 44] or [2, Section 23]). Note that here $f: G \rightarrow S$ is continuous iff $f$ is constant on the cosets of $G_{e}$.

THEOREM 6. Any A-space torsion topological group $G$ can be uniquely expressed as the extension of an indiscrete normal subgroup $N$ by a discrete group $D$. Furthermore if $D$ is finitely generated, $G^{*}$ is topologically isomorphic to $D$ made abelian. 
Proof. Clearly $N=G_{e}$ and $D=G / G_{e}$ gives such an extension. Conversely, if $1 \rightarrow N \rightarrow G \rightarrow D \rightarrow 1$ is exact and $D$ is discrete, $N$ must be open. Thus $G_{e} \subset N$. If $G_{e} \neq N$ then $N$ is not indiscrete, so $G_{e}=N$

The exactness of $G_{e} \rightarrow G \stackrel{p}{\longrightarrow} G / G_{e} \rightarrow 1$ gives the exactness of $I \rightarrow \operatorname{Hom}_{c}\left(G / G_{e}, S\right) \stackrel{p^{*}}{\longrightarrow} \operatorname{Hom}_{c}(G, S) \rightarrow \operatorname{Hom}_{c}\left(G_{e}, S\right)$ where $p^{*}$ is a continuous homomorphism. Since $G_{e}$ is indiscrete, $\operatorname{Hom}_{e}\left(G_{e}, S\right)=1$. Thus $p^{*}$ is a continuous isomorphism. Since $p$ is also a compact mapping, $p^{*}$ is an open mapping and thus is a topological isomorphism. If $D=G / G_{e}$ is finitely generated, $\operatorname{Hom}_{c}(D, S)$ is a discrete group topologically isomorphic to $\operatorname{Hom}(D, S)$. But since $S$ is abelian, $\operatorname{Hom}(D, S) \approx \operatorname{Hom}\left(D / D^{\prime}, S\right) \approx D / D^{\prime}$. Thus $G^{*}$ is topologically isomorphic to $D$ made abelian.

If $G$ is a Hausdorff abelian topological group, the duality theorem $[2,24.8]$ shows $G^{* *}$ is topologically isomorphic to $G$. This theorem is seen not to be true if Hausdorff separation is not required. In fact the following result shows how the topology on $G$ can be changed without changing $G^{*}$. Examples showing that the condition $G^{\prime} H=G^{\prime} K$ is not necessary are easy to construct.

THEOREM 7. Let $G_{1}$ and $G_{2}$ be the torsion group $G$ with the A-space topology determined by the normal subgroups $H$ and $K$ respectively. Then $G^{\prime} H=G^{\prime} K$ is a sufficient condition for $G_{1}^{*}$ to be topologically isomorphic to $G_{2}^{*}$.

Proof. The natural projection $p: D \rightarrow D / D^{\prime}$ gives a continuous isomorphism $p^{*}: \operatorname{Hom}_{c}\left(D / D^{\prime}, S\right) \rightarrow \operatorname{Hom}_{c}(D, S)$. The function $q^{*}: \operatorname{Hom}_{c}(D, S) \rightarrow \operatorname{Hom}_{c}\left(D / D^{\prime}, S\right)$ given by $q^{*}(f)\left(d D^{\prime}\right)=f(d)$ is well defined and the inverse to $p^{*}$. To show $q^{*}$ is continuous, consider the open set in $\operatorname{Hom}_{c}\left(D / D^{\prime}, S\right),\left(K D^{\prime}, V\right)=\left\{h: D / D^{\prime} \rightarrow S \mid h\left(K D^{\prime}\right) \subset V\right\}$ where $V$ is open in $S$ and $K D^{\prime}=\left\{k D^{\prime} \mid k \in K \subset D\right\}$ is compact and hence a finite set in $D / D^{\prime}$. Then $q^{*^{-1}}\left(K D^{\prime}, V\right)=(K, V)$ is a basic open set in 
$\operatorname{Hom}_{c}(D, S)$. So $q^{*}$ is continuous and $p^{*}$ is a homeomorphism. Thus $G_{1}^{*}$ and $G_{2}^{*}$ are topologically isomorphic to $\operatorname{Hom}\left(G / H /(G / H)^{\prime}, S\right)$ and $\operatorname{Hom}\left(G / K /(G / K)^{\prime}, S\right)$ respectively. However as discrete groups $G / H /(G / H)^{\prime}$ \& $G / H / G^{\prime} H / H \approx G / G^{\prime} H$. So if $G^{\prime} K=G^{\prime} H$ it follows that $G_{-1}^{*}$ is topologically isomorphic to $G_{2}^{*}$.

\section{References}

[1] P. Alexandroff, "Diskrete Räume", Rec. Math. Moscou (N.S.) 2 (1937), 501-518.

[2] Edwin Hewitt and Kenneth A. Ross, Abstract harmonic analysis, Vol. I (Die Grundlehren der mathematischen Wissenschaften, Band 115; Academic Press, New York; Springer-Verlag, Berlin, Göttingen, Heidelberg, 1963).

[3] Taqdir Husain, Introduction to topological groups (W.B. Saunders, Philadelphia, London, 1966).

[4] V. Krishnamurthy, "On the number of topologies on a finite set", Amer. Math. Monthly 73 (1966), 154-157.

[5] Michael C. MicCord, "Singular homology groups and homotopy groups of finite topological spaces", Duke Math. J. 33 (1966), 465-474.

[6] Sidney A. Morris and H.B. Thompson, "Topologies on finite groups", Bul2. Austral. Math. Soc. 1 (1969), 315-317.

[7] D. Stephen, "Topology on finite sets", Amer. Math. Monthly 75 (1968), 739-741.

[8] R.E. Stong, "Finite topological spaces", Trans. Amer. Math. Soc. 123 (1966), 325-340.

University of Nebraska,

Lincoln, Nebraska,

USA. 